\title{
Case Analysis of Misdiagnosis in Emergency Internal Medicine
}

\author{
Yuzhen $\mathrm{Hu}^{\mathbf{1}^{*}}$ and Liguo $\mathrm{Shi}^{2}$ \\ ${ }^{1}$ Department of Emergency, Xilin Gol League Hospital, Xilinguole, Inner Mongolia 026000, China \\ ${ }^{2}$ Inner Mongolia Blood Center, Xilin Gol League Hospital, Xilinguole, Inner Mongolia 026000, China
}

\begin{abstract}
Objective: This paper attempts to determine the clinical factors associated with the diagnosis of stroke in young adults. Method: We registered and examined young patients from the hospital stroke center. Demographic data, past medical history, 3 hours diagnosis time within the time frame, and the outcomes were assessed. We compared the patients with misdiagnosis and those who were correctly diagnosed in order to identify the factors associated with acute stroke. Results: From 2008 to 2014, 57 patients (16-50 years) were enrolled in the registry. 8 patients (14\%; 4 men and 4 women, mean age 38 years) were misdiagnosed. Of these 8 patients, seven were initially discharged from the emergency department. Patients aged less than 35 years old $(p=0.05)$ and posterior circulation stroke patients $(p=0.006)$ were more likely to be misdiagnosed. Conclusion: First aid department needs to popularize the awareness of stroke among young adults. Misdiagnosis may cause the patient to lose the best chance of thrombolysis.
\end{abstract}

\section{KEYWORDS}

Cerebral infarction

Misdiagnosis

Thrombolysi

\section{Introduction}

Stroke is the leading cause of adult disability and is a nervous system disorders that the doctor has the opportunity to completely cure. In the early stage of the treatment, the neurological function of patients with acute ischemic stroke can be recovered. As the result of stroke in the elderly was more common than the youth, the symptoms of the nervous system malfunctions that occurred in young people were often classified as other reasons. In addition, the cause of stroke in young people was also different from the elderly. Factors such as atherosclerosis, occlusion of atrial fibrillation (AF), and lacunar stroke often caused stroke in the elderly while less occurrence was found in young patients. Ischemic stroke was more common in young patients.

Copyright $\odot 2015$ Yuzhen $\mathrm{Hu}$ and Liquo Shi

doi: $10.18686 /$ aem.v4i4.10

Received: September 3, 2015; Accepted: November 10, 2015; Published online: December 30, 2015

This is an open-access article distributed under the terms of the Creative Commons Attribution Unported License (http://creativecommons.org/ licenses/by-nc/4.0/), which permits unrestricted use, distribution, and reproduction in any medium, provided the original work is properly cited.

${ }^{*}$ Corresponding author: Department of Emergency, Xilin Gol League Hospital, Xilinguole, Inner Mongolia 026000, China. E-mail: hu_yu_ zhen@sina.com
In this paper, we have listed a series of cases, in which, they were misdiagnosed for other diseases, and eventually diagnosed as ischemic stroke. We also explored the clinical characteristics associated with the diagnosis of stroke in young adults. To our concern, the factors associated with the diagnosis of stroke in young adults have not been reported elsewhere [1].

\section{Clinical data}

Data mining since 2006 at our comprehensive stroke center and clinic comprised of patients with aged 16-50 years old. In vascular risk factors, the patients possess a history of hypertension, diabetes, high blood cholesterol, smoking, and coronary artery bypass grafting or stenting. High blood pressure was being taken in the two random measurements with systolic blood pressure $>140 \mathrm{mmHg}$ or diastolic blood pressure $>90 \mathrm{~mm}$. Diabetes was being treated with anti-diabetic drugs with fasting blood glucose levels of $126 \mathrm{mg} / \mathrm{L}$. Hyperlipidemia patients refers to those who were being treated with cholesterol-lowering drugs with fasting total cholesterol levels $>200 \mathrm{mg} / \mathrm{L}$ or low density lipoprotein (LDL) cholesterol levels $>100 \mathrm{mg} / \mathrm{L}$.

\section{Statistical data analysis}

All statistical analysis were analyzed using SPSS 17.0, numerical variables said that categorical variables expressed 
as a percentage; between two averages were compared using $t$ test, two sample rate or composition compared by chi square test. Test level was set at $p<0.05$.

\section{Results}

Diagnosis of acute stroke patients were found to have the followings results, i.e., 4 cases diagnosed with peripheral vertigo, 1 case diagnosed with alcohol poisoning and was fully under hospital observation, 1 case diagnosed as migraine, 1 case of patient with lumbar puncture which has been ruled out as subarachnoid hemorrhage. Finally, 6 out of the 8 patients were diagnosed as ischemic stroke in the vertebral basilar artery. Out of 8 patients, 7 were delivered to the emergency department within 3 hours of the onset of symptoms. We judged that 3 patients could be considered as candidates for and 5 could be due to the fact that the symptoms were too mild to be considered as thrombolysis. Out of the 8 patients, two had a significant disability, which includes 4 patients with severe problem of ataxia and cognitive ability while 8 patients were diagnosed with aphasia.

Table 1 summarizes the clinical variables and their relationship to misdiagnosis rate. Both patients with posterior circulation stroke $(p=0.006)$ and $33 \%$ of patients aged more than 35 years old $(p=0.052)$ revealed a misdiagnosis rate of $38 \%$. There was no significant difference in terms of misdiagnosis based on gender, race, history of migraine, or any other traditional risk factors.

Table 1. Clinical variables and misdiagnosis rate.

\begin{tabular}{cccc} 
Category & $\begin{array}{c}\text { Correct diagnosis } \\
(\mathrm{n}=49)\end{array}$ & $\begin{array}{c}\text { Misdiagnosis } \\
(\mathrm{n}=8)\end{array}$ & $p$ \\
\hline Female & $30(88 \%)$ & $4(12 \%)$ & 0.70 \\
Male & $19(83 \%)$ & $4(17 \%)$ & \\
White & $34(84 \%)$ & $6(15 \%)$ & 1.0 \\
Black & $14(88 \%)$ & $2(12 \%)$ & \\
Age $>35$ & $41(91 \%)$ & $4(9 \%)$ & 0.052 \\
Age $<35$ & $8(67 \%)$ & $4(33 \%)$ & \\
Anterior circulation & $39(95 \%)$ & $2(5 \%)$ & 0.006 \\
Post cycle & $10(63 \%)$ & $6(38 \%)$ & \\
Migraine & $8(89 \%)$ & $1(11 \%)$ & 1.0 \\
No migraine & $41(85 \%)$ & $7(15 \%)$ & \\
\hline
\end{tabular}

\section{Discussion}

Misdiagnosis is a condition arose due to the misjudgment of medical diagnosis by medical doctors or the occurrence of medical malpractice that worsened the patient's condition and may even die. Misdiagnosis is the main factor leads to the tension and argument between doctors and patients, which also proof to be harmful to the patients' families. There are many factors to be considered in the emergency internal medicine which leads to misdiagnosis namely, the medical equipment, disease and physicians themselves. Lacking of experiences by the medical doctors and having less knowledge pertaining to the problem were the major causes of misdiagnosis that eventually affecting the normal medical judgement. For instance, doctors have not thoroughly gone insight into the symptoms and characteristics of different diseases [2-4]. At present, there is a great need for doctors to perform device calibration and compare the results of the device with a reference or standard in order determine the patient's condition. By right, this is another important factor that contributes to medical misdiagnosis with such cases accounted for $56 \%$ from the reported statistics. The other main factors of misdiagnosis were that the clinical symptoms of many diseases were not obviously seen and detected. Many new diseases begin to overflow due to the mankind advancement, therefore the original disease also began to change gradually. Subsequently, this event provide to the misunderstanding of the native characteristics of the disease that mislead the diagnosis process and delaying the treatment. Due to the high rate of misdiagnosis, many patients lose their quality lives and even leave their legacy behind. This kind of misdiagnosis has to stop in order to avoid medical accident which is definitely affecting the image of the hospital to the public. From the research point of view, a lot of patients missed the best treatment due to the early misdiagnosis and lead to certain illness that the consequences were selfevident. For such an event, the hospitals should have prevention awareness in order to prevent misdiagnosis of the illness again. By right, doctors and physicians should study in-depth pertaining to the diseases since they were the direct fuse of misdiagnosis due to the reason of having less knowledge and experiences. Additionally, these medical practitioners must be open-minded to ask the predecessors for several help in order to improve their professionalism. Besides that, patients should get consultation from medical doctor or specialist in order to dispel the idea of facing fear as part of life, to love and respect for science, to view life as a whole and to solve difficult problems in a timely manner. In order to avoid arbitrary, reckless and so on, the diagnosis must be based on medical history as well as physical and auxiliary examinations. Moreover, medical practitioners must have initiatives such as communicating with experienced personnel, sorting out their work and ideas so that the indirect lessons could be learned and eventually improve the diagnostic practice skills. Hospital's equipment should be upgraded in a timely manner since the cause of many diseases is very complex. Furthermore, doctors need to rely on the clinical data generated from the equipment in order to determine the condition of the disease. One of the important reasons for misdiagnosis was related to medical equipment failures which resulted in inaccurate examination results. In order to avoid the misdiagnosis by the medical equipment and to ensure the clinical examination results are correct, the hospital should calibrate the medical equipment on time [5]. In the process 
of diagnosis, physicians need to adjust the suitable timebased consultation for the patients in order to understand their physical condition and provide patients with clearer view pertaining to their disease with the aid of the medical equipment. Diagnosis need to be carried out cautiously and repeatedly to be confirmed as true after each diagnosis on the condition of fully grasp and by all means looking at a comprehensive view of the disease. Effective time of communication with the patient to understand the medical history besides the emergency internal medicine was another important part of the auxiliary diagnosis.

In the present paper, 8 patients with misdiagnosis occurrence were not diagnosed by the neurological department of internal medicine. In a prospective study conducted by Moulin et al., the analysis of the impact of the experts on the patient's diagnosis from the department of internal medicine showed that dizziness was the most common symptom of misdiagnosis to stroke. $37.3 \%$ of false positive and $36.6 \%$ of false negative diagnosis were discovered in 1679 patients from the diagnosis results of both the nervous system emergency and neurological department of internal medicine team, respectively [6].

Diagnosis of dizziness by nervous system emergency physicians was not practical. Based on investigation [5], only $3.2 \%$ out of 1666 patients who was diagnosed with vertigo limb symptoms and dizziness showed stroke or TIA. A survey was conducted in 13 out of 9472 patients in the case entitled for emergency patients, the true cause of dizziness due to the hardening of cerebral blood vessels was accounted for only $4 \%$ in total.

The consequences of misdiagnosis could be severe, especially for the cerebellum/brainstem infarction. In the case of a recurrence, the total mortality rate of these patients was $40 \%$, while for the survivors with no symptoms the total mortality rate was $50 \%$. This study suggested that an error in diagnosis of cerebellar infarction could result in severe morbidity and mortality. Emergency physicians should understand that computer tomography
(CT) is extremely low in sensitivity for the identification of a greater stroke. Finally, since our registry consisted of only young adult patients, we were unable to deduce on the misdiagnosis rate of stroke patients in the whole population [7].

In short, the emergency treatment of acute stroke patients may have serious consequences including long-term disability. It is important to raise the awareness of stroke in young adults found in the emergency department by the medical staffs and doctors. The differences in diagnostic accuracy between certified primary stroke center and noncertified hospital should also be resolved.

\section{Conflicts of interest}

These authors have no conflicts of interest to declare.

\section{Authors' contributions}

These authors contributed equally to this work.

\section{References}

1. American Heart Association. Heart disease and strokestatistics: 2009 update. Circulation. 2009;119:1-161.

2. Saver JL. Time is brain-quantified. Stroke. 2006;37:263-266.

3. Putaala J, Metso AJ, Metso TN. Analysis of 1008 consecutive patients aged 15 to 49 with first-ever ischemicstroke: The Helsinki Young Stroke Registry. Stroke. 2009;40:1195-1203.

4. Goldstein LB, Matcher DB. Clinical assessment of stroke. JAMA. 1994;271:1114-1120.

5. Ma HQ. Analysis and exploration of the causes of misdiagnosis in emergency internal medicine and explore the. Chinese Health Industry. 2013;(3):16.

6. Zhou F, Zhou MH, Yong M. Emergency medicine: 13 cases were misdiagnosed reasons analysis. Health Reading. $2011 ;(6): 210$.

7. Li YL, Liu CX. Analysis of the causes of misdiagnosis in 35 cases. Chinese Traditional Chinese Medicine. 2009;7(11):111. 